\title{
Morphometric Analysis of Suprarenal Glands (Adrenal Glands) with Multislice Computerized Tomography
}

\author{
Análisis Morfométrico de Glándulas Suprarenales (Glándulas Adrenales) \\ con Tomografía Computarizada Multicorte
}

\begin{abstract}
Duygu Akin¹; Mehmet Tugrul Yilmaz; Orhan Ozbek²; Hüseyin Özbiner³; Aynur Emine Cicekcibasi ${ }^{1}$; Mustafa Büyükmumcu ${ }^{1}$ \& Anil Didem Kabakci ${ }^{1}$
\end{abstract}

AKIN, D.; YILMAZ, T. M.; OZBEK, O. ; ÖZBINER, H.; CICEKCIBASI, A. E.; BÜYÜKMUMCU, M. \& KABAKCI, A. D. Morphometric analysis of suprarenal glands (adrenal glands) with multislice computerized tomography. Int. J. Morphol., 35(1):120-127, 2017.

SUMMARY: The aim of our study are to determine the shape, vertebrae level, height, volume, corpus medial and lateral branch thicknesses of AG and the distance from some neighboring structures on multidetector computed tomography (MDCT) images and also to determine the relationship between these data and gender, age, height, weight and body mass index (BMI). The study was conducted on a total of 420 MDCT images consisted of 220 male (mean age 63.44 $\pm 8.00,50-81$ years), 200 female (mean age 61.23 $\pm 7.95,50-84$ years) healthy individuals. Vertebrae level of AG (VLAG), height of AG (HAG), adrenal gland volume (VAG), corpus thickness of AG (CTAG), medial section thickness of AG (MTAG) and lateral section thickness of AG (LTAG) were investigated. In our study, RAG were observed on T12 vertebrae level in $38.19 \%$ of males and $42.5 \%$ of females and LAG were also observed at that level in $39.55 \%$ of the male and $51 \%$ of the female individuals. RAG were observed in all males and $98.5 \%$ of the females and LAG were observed $88.63 \%$ of the males and $94 \%$ of the females "Y" shaped. VAG were observed lower in males compared to females in age groups and VAG was observed increasing with age in males; decreased in 60-69 age group of females and increased again in 70 and plus age group. The aim of this study is to determine normal ranges of AG sizes and the relationship of each one with sex, age, height, weight and BMI.

KEY WORDS: Adrenal volume; Multidetector computed tomography (MDCT); Adrenal gland shape.

\section{INTRODUCTION}

Adrenal gland (AG) is a pair of endocrine gland which is mandatory for sustainability of life and function to ensure the general physiological organization of the organism. AG was mentioned for the first time in 1563 by Bartolomeo Eusthachia (Gaunt, 1975).

AG also being known as adrenal, suprarenal or surrenal gland is just due to its localizations. They are not related to the kidneys functionally. They are observed yellow-brown colored due to the lipoid matter they contain (Moore \& Persaud, 1993; Anand \& Vijayan; 1998, Avisse et al., 2000).

AG are triangular like shaped endocrine glands located on the posterior wall of the abdomen with kidneys at L1 vertebrae level and on antero-superior of the upper end of each kidney (Baxter \& Tyrrell, 1981; Harrison, 1985;
Moore \& Persaud; Anand \& Vijayan; Avisse et al.). Normally, weight of each adrenal gland varies between 3, 5 to 6 grams in adults (Tannenbaum 1975; Moore \& Persaud; Anand \& Vijayan; Avisse et al.). Right adrenal gland (RAG) is a pyramid shaped gland which is located close to inferior vena cava (IVC) and is in strong contact with uncovered area of the liver. Left adrenal gland (LAG) is semilunar shaped and slightly longer and wider compared to the right one. It is neighbored by abdominal aorta (AA) and the cardia of the stomach. Lower part of the anterior face, which is not covered by peritoneum, is in contact with pancreas, splenic artery and partially with spleen.

Shape and volume of the organs can change due to many diseases. To detect these changes, normal anatomical ranges must be known initially (Konus et al., 1998).

\footnotetext{
${ }^{1}$ Department of Anatomy, Meram Faculty of Medicine, Necmettin Erbakan University, Konya, Turkey.

${ }^{2}$ Department of Radiology, Meram Faculty of Medicine, Necmettin Erbakan University, Konya, Turkey.

${ }^{3}$ Department of Radiology, Faculty of Medicine, Selcuk University, Konya, Turkey.
} 
Therefore it is important to determine the pathological changes of $\mathrm{AG}$ sizes in adult individuals. To understand the pathological changes, normal ranges must be known.

In this study, we aimed to determine the shape of AG, vertebrae level, height, volume, thickness of corpus, medial and lateral branches with the distance to vertebral column, esophagus, inferior vena cava, aorta and diaphragm on Multidetector Computed Tomography (MDCT) images and to assess the relation of these parameters with sex, age, height, weight and BMI.

\section{MATERIAL AND METHOD}

To assess and measure AG type, thickness, volume and distance from anatomical structures, Necmettin Erbakan University, Meram Medical Faculty Radiology Department archives were scanned for 1000 individuals (500 female, 500 male) that had MDCT imaging for any reason retrospectively. All patients with adrenal gland mass, with mass other than AG, endocrine disorders which could cause AG hyperplasia, high levels of adrenocorticotrophic hormone, patients with any reason to complicate $\mathrm{AG}$ assessment on images, such as the ones with inadequate imaging technique or movement during imaging, and those in which lateral branch of the RAG were not observed clearly due to liver vicinage were excluded from the study. The study was conducted on MDCT images of 220 healthy males (mean age $63.44 \pm 8.00,50-81$ years) and 200 healthy females (mean age 61.23 $\pm 7.95,50-84$ years). Measurements were performed by the same person and measurements were done using $\mathrm{kg}, \mathrm{cm}$ and $\mathrm{cm} 3$ as units of measurement. Necessary permissions for the study were obtained from Necmettin Erbakan University Meram Medical
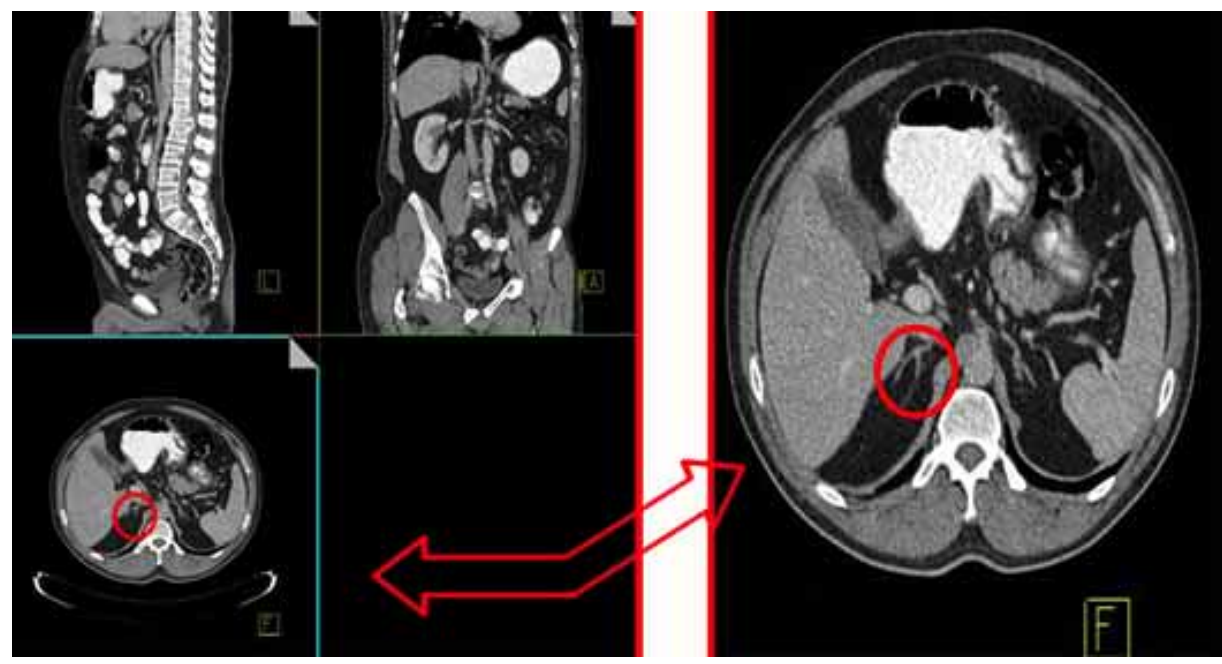

Fig. 1. AG shape
Faculty, Non-invasive clinical research Ethic Board with conclusion number 2012/50. Data regarding to age, weight, height and BMI values of the patients is shown in Table I.

64 channel MDCT device (Somatom Sensation 64, Siemens, Erlangen Germany) was used for the study. Patients were placed with a 22 Gauge branule to their visible forearm veins and total of $100 \mathrm{cc}$ contrast matter was given 3-4 cc per second. Abdomen MDCT images were captured in portal phase (60-65 seconds after the initialization of contrast matter) according to these parameters; KV: 120, MAS:86, Effective MaS: 50-170, Detector space: $1.2 \mathrm{~mm}$, Slice thickness: 1,5 mm, Pitch: 1.4 , rotation speed: $0.5 \mathrm{~s}$.

Measurements related to adrenal gland: AG shape: Typing of the AG was done according to the data on literature after detection of $\mathrm{AG}$ on axial reformat images (Fig.1).

Vertebrae level of AG (VLAG): The level of AG according to vertebrae was determined on coronal images after the AG was detected on axial reformat images (Fig. 2).

Height of AG (HAG), Adrenal gland volume (VAG): height and volume (VAG) measurements of AG was performed for each AG with Volume ${ }^{\circledR}$ software by using Leonardo Workstation. Axial and coronal plain measurement circles were determined as containing AG and lipoid tissue around the AG for HAG measurements and Volume ${ }^{\circledR}$ software was used to calculate the height of AG (Fig. 3).

Corpus thickness of AG (CTAG), medial section thickness of AG (MTAG), lateral section thickness of AG (LTAG): After the detection of AG on axial reformat images; corpus, medial and lateral branch thickness measurements were performed. After the detection of medial and lateral branches of AG in axial images, corpus measurements were done in transverse plain (Figs. 4.A-C; 5.A-B; 6.A-C).

Measurements of $\mathrm{AG}$ distance to neighboring structures: After the detection of AG on axial reformat images, the distances between $A G$ and esophagus (ODAG), vertebral column (VDAG), inferior vena cava (IVCDAG), abdominal aorta (AADAG) and diaphragm (DDAG) were measured. Also the individuals were assessed in 3 decades as 50-59, 60-69 and 70 and plus (Figs. 5.A-B; 6.A-C). 
Table I The demographic data of cases ( $n=$ Total number of cases, Mean \pm SD: mean value \pm standard deviation, p: Significance level).

\begin{tabular}{lllcc}
\hline & SEX & n & Mean \pm SD & p \\
\hline \multirow{2}{*}{ AGE } & Male & 220 & $63.44 \pm 8.00$ & \multirow{2}{*}{$0.005^{*}$} \\
& Female & 200 & $61.23 \pm 7.95$ & \\
HEIGHT $(\mathrm{cm})$ & Male & 220 & $1.70 \pm 6.34$ & $0.000^{*}$ \\
& Female & 200 & $1.60 \pm 0.06$ & \\
\multirow{2}{*}{ WEIGHT $(\mathrm{kg})$} & Male & 220 & $74.17 \pm 12.77$ & \multirow{2}{*}{0.756} \\
& Female & 200 & $75.00 \pm 12.58$ & \\
BMI $\left(\mathrm{kg} / \mathrm{m}^{2}\right)$ & Male & 220 & $25.65 \pm 3.92$ & \multirow{2}{*}{$0.000^{*}$} \\
& Female & 200 & $29.48 \pm 5.02$ & \\
\hline
\end{tabular}

*Statistically significant difference found using $\mathrm{p}<0.05$

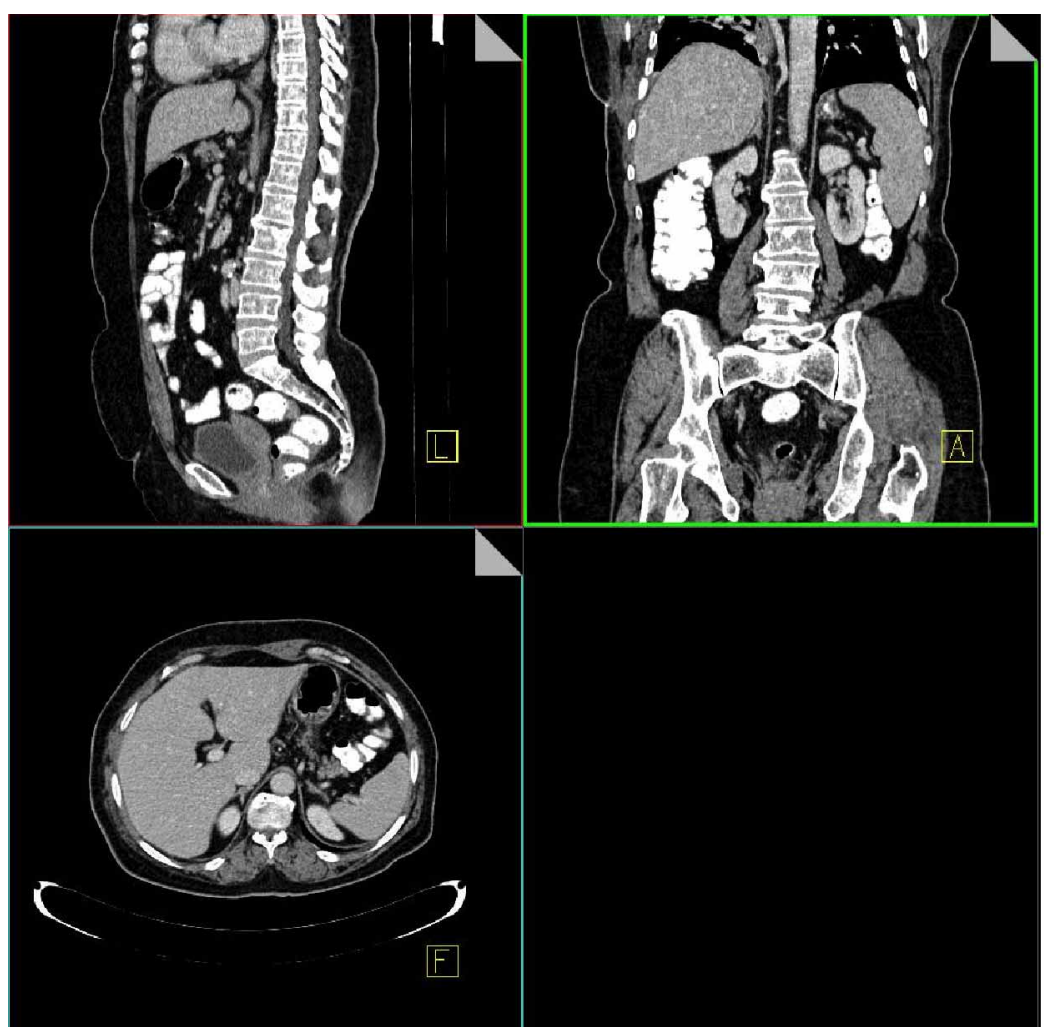

Fig. 2. Axial reformatted image on the level of AG according to the vertebral column
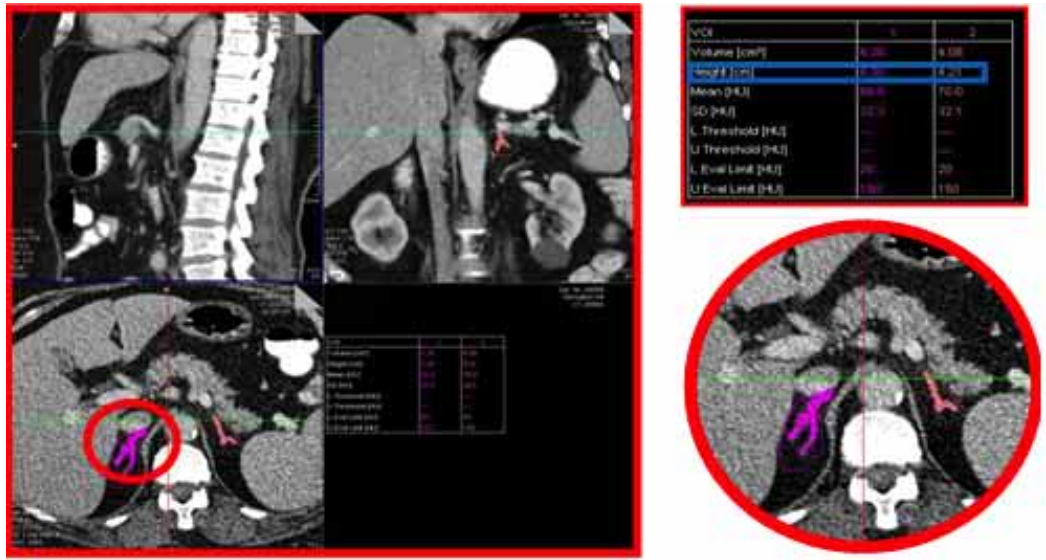

Fig. 3. Height of AG ( HAG) and adrenal gland volume (VAG)
Data obtained from the study was assessed using SPSS 15.0 (Statistical Package for Social Sciences) program. Definitive measurements of all variables were calculated. Quantitative variables were processed as \pm standard deviation; categorical variables were assessed as frequency and percent rates. Quantitative variables were checked as if they fit in normal distribution by using Kolmogrov Smirnov and Shapino-Wilk tests. Differences between male and female AG morphometric measurements and the differences between right and left side measurements were compared by using independent sample t-test and paired sample t-test, respectively. Also, the correlation values of $\mathrm{Ag}$ measurements and age, weight, height and BMI were determined with Pearson correlation test. The relation of BMI with VAG was formulized by using linear regression analysis. $\mathrm{P}<0.05$ value was considered statistically significant for all analysis.

\section{RESULTS}

RAG were observed in all males and $98.5 \%$ of females and LAG were $88.63 \%$ of the males and $94 \%$ of the females "Y" shaped in our study (Table II, Fig. 1).

RAG were observed in $38.19 \%$ of the males and $42.5 \%$ of the females and LAG were $39.55 \%$ of the males and $51 \%$ of the females on T12 vertebrae level (Table III).

A statistically significant relationship was observed in all parameters when parameters were compared between males and females $(\mathrm{P}<0.05)$ except right $\mathrm{CTAG}$ and IVCDAG; left HAG, VAG, CTAG, LTAG and VDAG values (Table IV).

When VDAG, ODAG, IVCCAG, AADAG and DDAG values were compared between males and females in age groups a significant relationship $(\mathrm{p}<0.05)$ was found between right and left side in all individuals and age groups. Significant relationships $(\mathrm{p}<0.05)$ were observed between right and left VAG and HAG values of 50-59 age group males, 60-69 age group females between right 
and left VAG and 60-69 age group males between right and left MTAG values. VAG was observed lower in females compared to males in all age groups, increasing with age in males and decreased in female 60-69 age group and increased again in 70 and plus age group of females (Table V).

Correlation coefficients of male and female individuals' right and left side parameters and age, height, weight and BMI is given in Table 6 separately.

Negative correlations were found in all parameters with the exception of AADAG and DDAG of females and

Table II. The shape of the adrenal gland (n: Number of individuals).

\begin{tabular}{llcccc}
\hline SEX & The shape AG & \multicolumn{2}{c}{ Right } & \multicolumn{2}{c}{ Left } \\
& & $\mathbf{n}$ & $\mathbf{\%}$ & $\mathbf{n}$ & $\mathbf{\%}$ \\
\hline Male & L-type & & & 2 & 0.91 \\
$\mathbf{n = 2 2 0}$ & Y-type & 220 & 100 & 195 & 88.63 \\
& Triangular & & & 22 & 10 \\
& V-type & & & 1 & 0.46 \\
Female & L-type & 1 & 0.5 & & 0 \\
$\mathbf{n = 2 0 0}$ & Y-type & 197 & 98.5 & 188 & 94 \\
& Triangular & 2 & 1 & 12 & 6 \\
& v-type & & & & 0 \\
\hline
\end{tabular}

in males between both right and left ODAG, IVCDAG, AADGA and age.

Right-left VAG, IVCDAG and AADGA values were found in relation with weight in males as no such relation was observed in females. In male individuals, right and left IVCDAG value and weight and height were found related and there was a negative but weak relation with age.

Right and left VAG reference range related to BMI in males and females were determined using least squares regression analysis.

Male right-left VAG $(\mathrm{cm} 3)=-5.316+\mathrm{BMI}$ x0.160 Female RVAG $(\mathrm{cm} 3)=5.786-$ BMI x0.68 Female LVAG $(\mathrm{cm} 3)=6.598-\mathrm{BMI} x 0.063$

$47.6 \%$ of the all individuals included to the study were females and $52.4 \%$ were males. HAG, VAG, CTAG, MTAG, LTAG, VDAG, ODAG, IVCDAG, AADGA and DDAG values were measured on males and females in this study. Mean values and standard deviation values of the parameters were determined (Table IV and V).

Table III. Volume of RAG and LAG according to sex.

\begin{tabular}{lcccccccc}
\hline & $\begin{array}{c}\text { Male } \\
\text { Right }\end{array}$ & \multicolumn{3}{c}{ Left } & \multicolumn{3}{c}{$\begin{array}{c}\text { Female } \\
\text { Right }\end{array}$} & \multicolumn{3}{c}{ Left } \\
\hline $\mathbf{T}_{\mathbf{1 1}}$ & $\mathbf{n}$ & $\mathbf{\%}$ & $\mathbf{n}$ & $\mathbf{\%}$ & $\mathbf{n}$ & $\mathbf{\%}$ & $\mathbf{n}$ & $\mathbf{\%}$ \\
$\mathbf{T}_{\mathbf{1 1}}-\mathbf{T}_{\mathbf{1 2}}$ & 15 & 6.82 & 11 & 5 & 15 & 7.5 & 8 & 4 \\
$\mathbf{T}_{\mathbf{1 2}}$ & 84 & 8.64 & 10 & 4.55 & 26 & 13 & 19 & 9.5 \\
$\mathbf{T}_{\mathbf{1 2}}-\mathbf{L}_{\mathbf{1}}$ & 39 & 17.73 & 35 & 15.91 & 41 & 20.5 & 35 & 17.5 \\
$\mathbf{L}_{\mathbf{1}}$ & 57 & 25.91 & 72 & 32.73 & 30 & 15 & 30 & 15 \\
$\mathbf{L}_{\mathbf{1}}-\mathbf{L}_{\mathbf{2}}$ & 4 & 1.82 & 3 & 1.37 & 3 & 1.5 & 6 & 3 \\
$\mathbf{L}_{\mathbf{2}}$ & 2 & 0.91 & 2 & 0.91 & & 0 & & 0 \\
$\mathbf{T o t a l}$ & 220 & 100 & 220 & 100 & 200 & 100 & 200 & 100 \\
\hline
\end{tabular}

Table IV. Mean values and significance level of AG parameters in males and females ( p: Significance level).

\begin{tabular}{|c|c|c|c|c|c|c|c|c|c|c|c|}
\hline \multicolumn{3}{|c|}{ MALE } & \multicolumn{3}{|c|}{ FEMALE } & \multicolumn{3}{|c|}{ Male-Female } & \multicolumn{3}{|c|}{ Total individual } \\
\hline PARAMETER & Right & Left & $\mathbf{P}$ & Right & Left & $\mathbf{P}$ & RightP & LeftP & Right & Mean \pm SD & $\mathbf{P}$ \\
\hline HAG & $3.71 \pm 0.83$ & $2.91 \pm 0.79$ & $0.033^{*}$ & $3.47 \pm 0.96$ & $2.40 \pm 0.73$ & 0.203 & 0.007 & 0.086 & $3.60 \pm 0.90$ & $3.57 \pm 0.90$ & 0.536 \\
\hline VAG & $3.00 \pm 0.96$ & $3.47 \pm 1.25$ & 0.026 & $3.38 \pm 1.24$ & $3.44 \pm 1.20$ & $0.013^{*}$ & 0.001 & 0.111 & $3.18 \pm 1.12$ & $3.00 \pm 1.12$ & $0.001 *$ \\
\hline CTAG & $0.56 \pm 0.16$ & $0.57 \pm 0.23$ & 0.728 & $0.58 \pm 0.20$ & $0.58 \pm 0.21$ & 0.523 & 0.440 & 0.207 & $0.57 \pm 0.18$ & $0.58 \pm 0.22$ & 0.503 \\
\hline MTAG & $0.43 \pm 0.11$ & $0.47 \pm 0.10$ & $0.000^{*}$ & $0.41 \pm 0.10$ & $0.43 \pm 0.10$ & $0.010^{*}$ & 0.029 & 0.999 & $0.42 \pm 0.10$ & $0.45 \pm 0.10$ & $0.000^{*}$ \\
\hline LTAG & $0.40 \pm 0.11$ & $0.41 \pm 0$ & 0.462 & $0.38 \pm 0.10$ & $0.39 \pm$ & 0.165 & 0.038 & 0.252 & $0.39 \pm 0.11$ & 0.40 & 0.136 \\
\hline VDAG & $2.54 \pm 0.89$ & $2.91 \pm 0.79$ & $0.000^{*}$ & $1.84 \pm 0.61$ & $2.40 \pm 0.73$ & $0.000 *$ & 0.000 & 0.084 & $2.21 \pm 0.84$ & $2.67 \pm 0.80$ & $0.001 *$ \\
\hline ODAGO & $4.92 \pm 1.02$ & $3.47 \pm 1.25$ & $0.000^{*}$ & $5.40 \pm 1.11$ & $3.44 \pm 1.20$ & $0.000 *$ & 0.000 & $0.000^{*}$ & $5.15 \pm 1.09$ & $3.45 \pm 1.23$ & $0.000^{*}$ \\
\hline IVCDAG & $0.20 \pm 0.16$ & $5.03 \pm 0.87$ & $0.000^{*}$ & $0.21 \pm 0.21$ & $4.28 \pm 0.62$ & $0.000^{*}$ & 0.450 & $0.000^{*}$ & $0.21 \pm 0.18$ & $4.68 \pm 0.85$ & $0.000^{*}$ \\
\hline AADGA & $2.65 \pm 0.78$ & $1.65 \pm 0.52$ & $0.000^{*}$ & $2.28 \pm 0.62$ & $1.25 \pm 0.46$ & $0.000^{*}$ & 0.000 & $0.000^{*}$ & $2.47 \pm 0.73$ & $1.46 \pm 0.53$ & $0.000 *$ \\
\hline DDAG & $0.89 \pm 0.39$ & $1.20 \pm 0.50$ & $0.000 *$ & $0.80 \pm 0.38$ & $0.96 \pm 0.41$ & $0.000 *$ & 0.019 & $0.000 *$ & $0.84 \pm 0.39$ & $1.09 \pm 048$ & $0.000 *$ \\
\hline
\end{tabular}

*Statistically significant difference found using $\mathrm{p}<0.05$ 
Table V According to age groups Mean values and significance level of RAG and LAG parameters in males and females (n: Number of individuals, p: significance level).).

\begin{tabular}{|c|c|c|c|c|c|c|c|c|c|}
\hline \multirow[b]{2}{*}{ PARAMETER } & & \multicolumn{4}{|c|}{ Male } & \multicolumn{4}{|c|}{ Female } \\
\hline & & $\mathbf{n}$ & Right & Left & $\mathbf{P}$ & $\mathbf{n}$ & Right & Left & $\mathbf{P}$ \\
\hline \multirow{5}{*}{ HAG } & 50-59 age group & 70 & $3.82 \pm 091$ & $3.51 \pm 0.97$ & $0.015^{*}$ & 89 & $3.48 \pm 089$ & $3.66 \pm 092$ & 0.096 \\
\hline & 60-69 age group & 93 & $3.57 \pm 0.80$ & $3.52 \pm 0.77$ & 0.642 & 76 & $3.51 \pm 0.96$ & $3.46 \pm 1.00$ & 0.671 \\
\hline & 70 and over age group & 57 & $3.82 \pm 0.77$ & $3.73 \pm 0.88$ & 0.529 & 35 & $3.35 \pm 1.12$ & $3.52 \pm 0.76$ & 0.308 \\
\hline & 50-59 age group & 70 & $2.86 \pm 0.90$ & $2.68 \pm 0.88$ & 0.026 & 89 & $3.47 \pm 1.18$ & $3.31 \pm 1.11$ & 0.240 \\
\hline & 60-69 age group & 93 & $3.00 \pm 0.91$ & $2.91 \pm 1.09$ & 0.425 & 76 & $3.28 \pm 1.23$ & $3.01 \pm 1.18$ & 0.038 \\
\hline \multirow[t]{3}{*}{ VAG } & 70 and over age group & 57 & $3.19 \pm 1.09$ & $3.01 \pm 1.23$ & 0.210 & 35 & $3.35 \pm 1.42$ & $3.09 \pm 1.18$ & 0.297 \\
\hline & 50-59 age group & 70 & $0.53 \pm 0.14$ & $0.59 \pm 0.24$ & 0.058 & 89 & $0.58 \pm 0.21$ & $0.59 \pm 0.21$ & 0.468 \\
\hline & 60-69 age group & 93 & $0.57 \pm 0.16$ & $0.57 \pm 0.22$ & 0.920 & 76 & $0.58 \pm 0.21$ & $0.59 \pm 0.21$ & 0.779 \\
\hline \multirow[t]{3}{*}{ CTAG } & 70 and over age group & 57 & $0.58 \pm 0.17$ & $0.54 \pm 22$ & 0.229 & 35 & $0.55 \pm 0.17$ & $0.55 \pm 0.20$ & 0.943 \\
\hline & 50-59 age group & 70 & $0.44 \pm 0.11$ & $0.46 \pm 0.10$ & 0.157 & 89 & $0.42 \pm 0.11$ & $0.44 \pm 0.10$ & 0.070 \\
\hline & 60-69 age group & 93 & $0.42 \pm 0.10$ & $0.47 \pm 0.10$ & $0.000^{*}$ & 74 & $0.41 \pm 0.09$ & $0.43 \pm 0.11$ & 0.231 \\
\hline \multirow[t]{3}{*}{ MTAG } & 70 and over age group & 57 & $0.45 \pm 0.11$ & $0.46 \pm 0.11$ & 0.422 & 34 & $0.38 \pm 0.08$ & $0.41 \pm 0.07$ & 0.140 \\
\hline & 50-59 age group & 70 & $0.40 \pm 0.10$ & $0.41 \pm 0.10$ & 0.593 & 89 & $0.36 \pm 0.11$ & $0.39 \pm 0.12$ & 0.068 \\
\hline & 60-69 age group & 93 & $0.39 \pm 0.10$ & $0.41 \pm 0.11$ & 0.180 & 74 & $0.38 \pm 0.10$ & $0.39 \pm 0.11$ & 0.369 \\
\hline \multirow[t]{3}{*}{ LTAG } & 70 and over age group & 57 & $0.42 \pm 0.13$ & $0.40 \pm 0.12$ & 0.403 & 34 & $0.39 \pm 0.10$ & $0.37 \pm 0.08$ & 0.337 \\
\hline & $50-59$ yas grubu & 70 & $2.48 \pm 0.84$ & $2.86 \pm 0.83$ & $0.000^{*}$ & 89 & $1.73 \pm 0.52$ & $2.38 \pm 0.76$ & $0.000^{*}$ \\
\hline & 60-69 yas grubu & 93 & $2.56 \pm 0.94$ & $2.89 \pm 0.79$ & $0.002 *$ & 76 & $1.93 \pm 0.67$ & $2.54 \pm 0.68$ & $0.000^{*}$ \\
\hline \multirow[t]{3}{*}{ VDAG } & 70 ve üzeri ya_grubu & 57 & $2.59 \pm 0.88$ & $3.01 \pm 0.75$ & $0.000 *$ & 35 & $1.95 \pm 0.68$ & $2.16 \pm 0.72$ & $0.000 *$ \\
\hline & $50-59$ yas grubu & 70 & $4.89 \pm 1.06$ & $3.58 \pm 1.16$ & $0.000^{*}$ & 89 & $5.48 \pm 1.16$ & $3.48 \pm 1.24$ & $0.000^{*}$ \\
\hline & 60-69 yas grubu & 93 & $5.02 \pm 0.94$ & $3.37 \pm 1.26$ & $0.000 *$ & 76 & $5.31 \pm 1.03$ & $3.41 \pm 1.25$ & $0.000^{*}$ \\
\hline \multirow[t]{3}{*}{ ODAG } & 70 ve üzeri yas grubu & 57 & $4.79 \pm 1.11$ & $3.48 \pm 1.36$ & $0.000 *$ & 35 & $5.36 \pm 1.15$ & $3.38 \pm 1.03$ & $0.000 *$ \\
\hline & 50-59 yas grubu & 70 & $0.21 \pm 0.16$ & $4.98 \pm 0.74$ & $0.000 *$ & 89 & $0.19 \pm 0.21$ & $4.23 \pm 0.70$ & $0.000 *$ \\
\hline & 60-69 yas grubu & 93 & $0.19 \pm 0.15$ & $5.08 \pm 0.93$ & $0.000^{*}$ & 76 & $0.19 \pm 0.21$ & $4.23 \pm 0.70$ & $0.000^{*}$ \\
\hline \multirow[t]{3}{*}{ IVCDAG } & 70 ve üzeri yas grubu & 57 & $0.22 \pm 0.16$ & $5.03 \pm 0.92$ & $0.000 *$ & 35 & $0.26 \pm 0.26$ & $4.44 \pm 0.61$ & $0.000 *$ \\
\hline & 50-59 yas grubu & 70 & $2.62 \pm 0.77$ & $1.64 \pm 10.45$ & $0.000 *$ & 89 & $2.32 \pm 0.66$ & $1.21 \pm 0.46$ & $0.000 *$ \\
\hline & 60-69 yas grubu & 93 & $2.76 \pm 0.76$ & $1.70 \pm 0.54$ & $0.000 *$ & 76 & $2.29 \pm 0.57$ & $1.27 \pm 0.48$ & $0.000 *$ \\
\hline \multirow[t]{3}{*}{ AADGA } & 70 ve üzeri yas grubu & 57 & $2.50 \pm 0.83$ & $1.60 \pm 0.56$ & $0.000 *$ & 35 & $2.16 \pm 0.35$ & $1.31 \pm 0.40$ & $0.000 *$ \\
\hline & 50-59 yas grubu & 70 & $0.87 \pm 0.34$ & $1.18 \pm 0.50$ & $0.000 *$ & 89 & $0.77 \pm 0.40$ & $0.95 \pm 0.40$ & $0.000 *$ \\
\hline & 60-69 yas grubu & 93 & $0.89 \pm 0.39$ & $1.20 \pm 0.52$ & $0.000 *$ & 76 & $0.72 \pm 0.25$ & $1.01 \pm 0.43$ & $0.000 *$ \\
\hline DDAG & 70 ve üzeri vas grubu & 57 & $0.90 \pm 0.44$ & $1.21 \pm 0.48$ & $0.000 *$ & 35 & $1.01 \pm 0.46$ & $0.87 \pm 0.41$ & $0.000 *$ \\
\hline
\end{tabular}

*Statistically significant difference found using $\mathrm{p}<0.05$

\section{DISCUSSION}

Hormones secreted by AG are glucocorticoid, mineralocorticoid and androgenous hormones. Epinephrine and norepinephrine hormones are secreted by AG medulla. Hypersecretion or deficiency of these hormones cause different clinical syndromes. Correct assessment of AG sizes is important for clinical diagnosis and treatment. Changes of organ size and morphology occur in many diseases. AG masses can present with very different clinic, radiologic and laboratory findings. Also AG masses arepathologies with high risk of malignancy. Preoperative definitive diagnosis is difficult with existing diagnostic methods at this time. Various sources had reported that AG masses coincided on 0.6-1-4\% of the patients had Ct scans for different reasons (Gagner et al., 1997). They had mentioned that normal or pathological AG could be shown with CT scans swiftly, reliably and effectively.
Thomas Addison had detected a condition presents with fatigue, hyperpigmentation and results with death in 1855 and described the condition as due to destruction of AG. AG plays a very important role on stress response of the organism. Any hormone synthesis or secretion deficiency in any level of hypothalamic-pituitary-adrenal system results with AG cortex hypo function. Adrenal insufficiency can present as primary, secondary or tertiary. AG insufficiency can lead to very different clinical manifestations. It can lead to death of the patient if diagnosis is delayed (Gaunt).

Although nonfunctional adrenocortical carcinomas can be observed in all age groups, they most frequently are observed between ages of 50-70 (Cullen, 1985; Montero et al., 1990). Latent AG carcinoma incidence increases with age. Mean age 
Table VI The correlations between all the data obtained and the demographic values based on gender (n: Total number of cases, $r$ :

Correlation, p: Significance level).

\begin{tabular}{|c|c|c|c|c|c|c|c|c|c|c|c|}
\hline \multirow{2}{*}{ PARAMETER } & & & \multirow[b]{2}{*}{$\mathbf{n}$} & \multicolumn{2}{|c|}{ AGE } & \multicolumn{2}{|c|}{ HEIGHT } & \multicolumn{2}{|c|}{ WEIGHT } & \multicolumn{2}{|c|}{ BMI } \\
\hline & & & & $\mathbf{r}$ & $\mathbf{P}$ & $\mathbf{r}$ & $\mathbf{P}$ & $\mathbf{r}$ & $\mathbf{P}$ & $\mathbf{r}$ & $\mathbf{P}$ \\
\hline \multirow{4}{*}{ HAG } & \multirow{2}{*}{ MALE } & Right & 220 & 0.03 & 0.714 & 0.78 & 0.249 & $0.185^{* *}$ & 0.006 & $0.170^{*}$ & 0.12 \\
\hline & & Left & 220 & 0.09 & 0.205 & 0.019 & 0.785 & 0.062 & 0.36 & 0.54 & 0.43 \\
\hline & \multirow{2}{*}{ FEMALE } & Right & 200 & -0.01 & 0.947 & 0.057 & 0.421 & 0.084 & 0.237 & 0.07 & 0.36 \\
\hline & & Left & 200 & -0.100 & 0.171 & 0.054 & 0.446 & 0.137 & 0.053 & 0.11 & 0.11 \\
\hline \multirow{4}{*}{ VAG } & \multirow{2}{*}{ MALE } & Right & 220 & 0.121 & 0.074 & -0.072 & 0.291 & $-0.180^{* *}$ & 0.007 & -0.167 & 0.013 \\
\hline & & Left & 220 & 0.072 & 0.286 & -0.049 & 0.473 & $-0.213^{* *}$ & 0.002 & $0.218^{* *}$ & 0.001 \\
\hline & \multirow{2}{*}{ FEMALE } & Right & 198 & 0.017 & 0.809 & 0.027 & 0.702 & 0.038 & 0.59 & 0.028 & 0.69 \\
\hline & & Left & 196 & -0.065 & 0.359 & 0.042 & 0.559 & -0.094 & 0.185 & -0.108 & 0.127 \\
\hline \multirow{4}{*}{ CTAG } & \multirow{2}{*}{ MALE } & Right & 220 & $0.143^{*}$ & 0.034 & 0.015 & 0.822 & 0.011 & 0.875 & 0.003 & 0.963 \\
\hline & & Left & 220 & -0.068 & 0.314 & -0.047 & 0.487 & -0.084 & 0.214 & -0.072 & 0.289 \\
\hline & \multirow{2}{*}{ FEMALE } & Right & 200 & 0.003 & 0.97 & 0.011 & 0.874 & -0.045 & 0.526 & -0.052 & 0.465 \\
\hline & & Left & 200 & -0.002 & 0.972 & -0.037 & 0.559 & -0.113 & 0.11 & -0.093 & 0.193 \\
\hline \multirow{4}{*}{ MTAG } & \multirow{2}{*}{ MALE } & Right & 220 & 0.062 & 0.36 & 0.064 & 0.344 & 0.105 & 0.119 & 0.089 & 0.19 \\
\hline & & Left & 220 & 0.01 & 0.894 & 0.092 & 0.201 & 0.061 & 0.395 & 0.028 & 0.697 \\
\hline & \multirow{2}{*}{ FEMALE } & Right & 197 & -0.051 & 0.482 & -0.004 & 0.95 & 0.046 & 0.522 & 0.05 & 0.484 \\
\hline & & Left & 197 & -0.069 & 0.349 & 0.052 & 0.477 & -0.015 & 0.835 & -0.036 & 0.622 \\
\hline \multirow{4}{*}{ LTAG } & \multirow{2}{*}{ MALE } & Right & 220 & 0.095 & 0.161 & -0.028 & 0.678 & 0.073 & 0.28 & 0.098 & 0.147 \\
\hline & & Left & 220 & -0.032 & 0.656 & 0.063 & 0.383 & 0.13 & 0.07 & 0.125 & 0.083 \\
\hline & \multirow{2}{*}{ FEMALE } & Right & 197 & 0.080 & 0.266 & -0.072 & 0.316 & 0.071 & 0.327 & 0.101 & 0.160 \\
\hline & & Left & 197 & -0.010 & 0.89 & 0.001 & 0.99 & -0.042 & 0.575 & -0.044 & 0.549 \\
\hline \multirow{4}{*}{ VDAG } & MALF & Right & 220 & 0.032 & 0.641 & 0.117 & 0.082 & $0.323^{* *}$ & 0.000 & $0.312^{* *}$ & 0.000 \\
\hline & MALE & Left & 220 & 0.095 & 0.159 & $0.147^{*}$ & 0.029 & $0.242^{* *}$ & 0.000 & $0.198^{* *}$ & 0.003 \\
\hline & FEMALE & Right & 200 & $0.154^{*}$ & 0.029 & -0.008 & 0.913 & 0.066 & 0.353 & 0.075 & 0.293 \\
\hline & FEMALE & Left & 200 & -0.590 & 0.405 & 0.035 & 0.625 & $0.18^{* *}$ & 0.009 & $0.168^{*}$ & 0.017 \\
\hline & & Right & 220 & -0.048 & 0.477 & 0.1 & 0.14 & $0.148^{*}$ & 0.029 & 0.117 & 0.084 \\
\hline ODAG & MALE & Left & 220 & -0.001 & 0.987 & 0.011 & 0.874 & 0.126 & 0.063 & $0.140^{*}$ & 0.038 \\
\hline & FEMALE & Right & 200 & -0.074 & 0.295 & -0.05 & 0.486 & -0.088 & 0.217 & -0.0622 & 0.38 \\
\hline & & Left & 200 & -0.043 & 0.549 & -0.008 & 0.911 & 0.032 & 0.656 & 0.035 & 0.625 \\
\hline & MALE & Right & 220 & -0.032 & 0.638 & $0.113 *$ & 0.095 & $0.139 *$ & 0.039 & 0.11 & 0.103 \\
\hline IVCDAG & MALE & Left & 220 & -0.007 & 0.918 & $0.153 *$ & 0.023 & $0.188^{* *}$ & 0.005 & $0.148^{*}$ & 0.028 \\
\hline & FEMALF & Right & 200 & -0.079 & 0.265 & -0.106 & 0.135 & -0.105 & 0.139 & -0.049 & 0.491 \\
\hline & & Left & 200 & $-0.164 *$ & 0.02 & -0.099 & 0.164 & 0.037 & 0.602 & 0.09 & 0.205 \\
\hline & MAIF & Right & 220 & -0.056 & 0.407 & 0.119 & 0.079 & $0.158^{*}$ & 0.019 & 0.122 & 0.071 \\
\hline AADGA & MALE & Left & 220 & -0.043 & 0.528 & $0.184^{* *}$ & 0.006 & $0.247^{* *}$ & 0.000 & $0.196^{* *}$ & 0.003 \\
\hline & FEMALE & Right & 200 & -0.025 & 0.727 & -0.077 & 0.280 & 0.023 & 0.75 & 0.053 & 0.457 \\
\hline & FEMALE & Left & 200 & 0.092 & 0.197 & 0.005 & 0.946 & 0.108 & 0.128 & 0.111 & 0.017 \\
\hline & MaLF & Right & 220 & 0.019 & 0.775 & $0.219^{* *}$ & 0.001 & $0.297^{* *}$ & 0.000 & $0.239 * *$ & 0.000 \\
\hline & & Left & 220 & -0.008 & 0.909 & $0.230^{* *}$ & 0.001 & $0.16^{*}$ & 0.014 & 0.080 & 0.236 \\
\hline DDAG & FFMAIF & Right & 198 & $-0.186^{* *}$ & 0.009 & 0.090 & 0.206 & $0.261^{* *}$ & 0.000 & $0.227^{* *}$ & 0.001 \\
\hline & FEMALE & Left & 196 & $0.203^{* *}$ & 0.004 & $-0.124 *$ & 0.081 & 0.062 & 0.383 & $0.123^{*}$ & 0.083 \\
\hline
\end{tabular}

*Correlation is significant at the 0.05 level (2- tailed)

$* *$ Correlation is significant at the 0.01 level (2- tailed)

value in our study was 63 (50-81) for males whereas 61 (5084 ) for females. This age group was preferred for the study due to incidence of AG diseases being higher in 50 and plus age group.

Montagne et al., (1978) studied a total of 60 individuals (33 male, 27 female) whose mean age was 56. They used
MDCT images for their study and detected RAG mostly shaped as "L" and LAG as "Y" and "V". Miekos (1979) reported 61 $\%$ of RAG they collected from 220 adult cadavers were pyramid shaped, $20 \%$ biretta, $9 \%$ sandglass, $5 \%$ semilunar shaped and left glands were $63 \%$ semilunar, $19 \%$ triangular, $11 \%$ elliptical shaped and $7 \%$ were shapeless. Superiority of our study to the study of Montagne et al., comes from the 
number of patients assessed being higher in our study and the patient data categorization as male and female. In our study RAG were observed shaped in males $100 \%$ as "Y" and LAG were $88.63 \%$ "Y" and $10 \%$ triangular. RAG were $98.5 \%$ shaped as "Y", $1 \%$ triangular, $0.5 \% \mathrm{~L}$ shaped in female patients and LAG were $94 \%$ "Y", $6 \%$ triangular.

There aren't enough studies and standardization to this day regarding to AG thickness. In a study related to this subject, CTAG was assessed and CTAG was observed only 1 of 47 cases as over $1 \mathrm{~cm}$. Hamidi et al., (2012) had reported in their study that both CTAG were under $1 \mathrm{~cm}$ value in all 100 cases they assessed and they reported this finding as compatible with the literature (Montagne, 1978; Karstaedt, 1978). In our study, we observed 10 cases ( 8 females and 2 males on RCTAG, 9 female and 1 male on LCTAG) with maximal CTAG value were over $1 \mathrm{~cm}$. Vincent et al., (1994) had reported CTAG as average of $6.1 \mathrm{~mm}$ on right and 7.9 mm on left; Hamidi et al., had reported RCTAG as average of $4.37 \mathrm{~mm}$, LCTAG as $4.29 \mathrm{~mm}$. In our study; RAG of the males were $5.6 \mathrm{~mm}$, LAG were $5.7 \mathrm{~mm}$ in average and in females $5.8 \mathrm{~mm}$ and $5.9 \mathrm{~mm}$, respectively. LCTAG values of our study were observed similar to the study of Hamidi et al., and RCTAG values were observed similar to the study of Vincent et al.

Vincent et al., had measured MTAG and LTAG respectively on right as a mean value of $2.8 \mathrm{~mm}, 2.8 \mathrm{~mm}$ and on left as 33.3 and $3 \mathrm{~mm}$. Hamidi et al., had observed 3.02 and $2.82 \mathrm{~mm}$ on the right and $3.46 \mathrm{~mm}$ and $3.05 \mathrm{~mm}$ on the left. In our study; on males, RMTAG and RAGL were respectively $4.3 \mathrm{~mm}, 4 \mathrm{~mm}$ on average and LMTAG and LLTAG4, 7 and $4 \mathrm{~mm}$. In females these values were $4 \mathrm{~mm}$, 3.8 for RMTAG and RLTAG respectively and $4.3 \mathrm{~mm}$ and $3.9 \mathrm{~mm}$ for LMTAG and LLTAG respectively. Hamidi et al., had detected significant differences on LTAG and MTAG. No significant difference was detected regarding to LTAG in our study whereas MTAG were observed significantly different in both sexs. MTAG and LTAG were not observed over $1 \mathrm{~cm}$ value. MTAG and LTAG were observed thicker on left sides compared to right in accordance with the study of Hamidi et al. We believe that the gland on the left side being thicker than the right is due to shape variations of LAG. In our study, we also interestingly found statistically significant differences between MTAG and LTAG (Table II). We believe that MTAG could be thinner due to the fact that AGL are neighbored by solid organs and this finding can lead the way for future researches with more crowded number of cases.

Hamidi et al., and Vincent et al., had not mentioned any value regarding to CTAG, MTAG and LTAG. We assessed the CTAG, MTAG and LTAG values of males and females separately with the relation between sexs.
LAG is slightly longer and wider and closer to AA compared to RAG (Baxter \& Tyrrell; Wells \& Santen, 1986). Not many studies regarding to RAADAG and LAADAG exist in the literature. In our study; RAADAG was found $2.47 \pm 0.73 \mathrm{~cm}$ and LAADAG was found $1.46 \pm 0.53 \mathrm{~cm}$ in average without discriminating sex. The difference was found highly statistically significant. Mean value of RAADAG was measured $2.65 \mathrm{~cm}$ in males and $2.28 \mathrm{~cm}$ in females. Mean value of LAADAG was detected as $1.65 \mathrm{~cm}$ in males and $1.25 \mathrm{~cm}$ in females. RAADAG and LAADAG measurements were observed higher in males compared to females. The findings we obtained were seen in concordance with the findings of Baxter \& Tyrrell, Wells \& Santen. In males; RAADAG and LAADAG were both observed moderately negatively correlated with age and positively but moderately correlated with height, weight and BMI.

Schneller et al. (2014), had found mean RVAG and LVAG values on 69 male individuals as $3.96 \mathrm{~cm}^{3}$ and 5.18 $\mathrm{cm}^{3}$ and $2.94 \mathrm{~cm}^{3}$ and $4.19 \mathrm{~cm}^{3}$ on 39 female individuals respectively. In our study, we found RVAG as $3.00 \mathrm{~cm}^{3}$ and LVAG as $2.86 \mathrm{~cm}^{3}$ in 220 males and observed same parameters as $3.38 \mathrm{~cm}^{3}$ and $3.16 \mathrm{~cm}^{3}$ in 200 female individuals respectively. While the study of Schneller et al., reported higher measurements for females compared to males, our study suggests the opposite as VAG values are higher in males compared to females.

There are some studies in the literature that contain RVAG and LVAG measurements without discriminating sexs. Schneller et al., studied 105 patients and reported RVAG as $3.61 \mathrm{~cm}^{3}$ and LVAG as $4.84 \mathrm{~cm}^{3}$ on average. Similarly Wang et al., (2012) studied 81 patients and reported the mean values of RVAG and LVAG as $4.26 \mathrm{~cm}^{3}$ and $4.23 \mathrm{~cm} 3$ respectively. We measured VAG on 420 individuals and observed the RVAg values as $3.18 \mathrm{~cm} 3$ and LVAG as $3.00 \mathrm{~cm} 3$ in average. While our study is similar to the study of Wang et al., as the LVAG value is higher than RVAG value, it differs from the study of Schneller et al., for the same reason.

Our study also differs from the study of Schneller et $a l$., as we had a greater study population and from the study of Wang et al., as we separated the data according to sex.

The aim of this study was to determine the normal range of $\mathrm{AG}$ sizes and to investigate their relationship with sex, age, weight, height and BMI each. Also the relation between BMI and VAG is also shown with the obtained formulations. Therefore we believe that we formed a reference range for MDCT images on AG deviations outside the normal ranges and for this reason, we also believe that this study could act as a reference guide for radiologists and surgeons of this area. 
Normal data for shape, vertebrae level, height, volume, corpus medial and lateral branch thicknesses of $\mathrm{AG}$ and the distance from some neighboring structures are provided. Sex, age, height, weight BMI have major impact on adrenal gland volume and linear dimensions.

There is a concordance between volumetric and linear assessment; however, volumetry is more reproducible.

AKIN, D.; YILMAZ, T. M.; OZBEK, O. ; ÖZBINER, H.; CICEKCIBASI, A. E.; BÜYÜKMUMCU, M. \& KABAKCI, A. D. A. Análisis morfométrico de glándulas suprarrenales (glándulas adrenales) con tomografía computarizada multicorte. Int. J. Morphol., 35(1):120-127, 2017.

RESUMEN: Los objetivos de nuestro estudio fueron determinar la forma, el nivel vertebral, la altura, el volumen, el grosor de las ramas medial y lateral del cuerpo de glándulas suprarrenales (GSR) y la distancia de algunas estructuras vecinas en las imágenes de tomografía computada multidetector (TCMD) y determinar la relación entre estos datos y sexo, edad, estatura, peso e índice de masa corporal (IMC). El estudio se realizó en un total de 420 imágenes TCMD de 220 hombres (edad media 63,44 $\pm 8,00,50-81$ años) y 200 mujeres (edad media $61,23 \pm 7,95,50$ 84 años) todos sanos. Se investigó el nivel de la GSR respecto a las vértebras, la altura de GRS (AGSR), el volumen de la glándula suprarrenal (VGSR), el grosor del cuerpo, el espesor de la sección mediana de GSR (SMGSR) y el grosor de la sección lateral de GSR (SLGSR). En nuestro estudio, se observó la GSR derecha a nivel de vértebras T12 en el 38,19\% de los hombres y en el 42,5 $\%$ de las mujeres y la GSR izquierda se observó a ese nivel en el $39,55 \%$ de los hombres y $51 \%$ de las mujeres. La GSR se presentó en forma de «Y» en el lado derecho en todos los hombres y en el $98,5 \%$ de las mujeres y en el lado izquierdo se observó en el $88,63 \%$ de los hombres y $94 \%$ de las mujeres. El VGSR era menor en los hombres en relación con las mujeres en los diferentes grupos de edad y aumentaba con la edad en los hombres; disminuyó en el grupo de 60-69 años de edad de las mujeres y aumentó nuevamente después de los 70 años.

PALABRAS CLAVE: Volumen suprarrenal; Tomografía computarizada multidetector (MDCT); Forma de la glándula suprarrenal.

\section{REFERENCES}

Anand, L. N. \& Vijayan, E. Studies on the effect of intratesticular administration of opioid peptides, naloxone or $\mathrm{N}$-acetyl beta-endorphin antiserum on some testicular parameters in rats. Indian J. Physiol. Pharmacol., 42(1):107-12, 1998.

Avisse, C.; Marcus, C.; Patey, M.; Ladam-Marcus, V.; Delattre, J. F. \& Flament, J. B. Surgical anatomy and embryology of the adrenal glands. Surg. Clin. North Am., 80(1):403-15, 2000.

Baxter, J. D. \& Tyrrell, J. B. The Adrenal Cortex in Endocrinology and Metabolism. In: Felig, P.; Baxter, J. D.; Broadus, A. E. \& Frohman, L. A. (Eds.). Endocrinology and Metabolism. New York, McGraw Hill
Book Co., 1981. pp.385.

Cullen, M. L.; Staren, E. D.; Straus, A. K.; Doolas, A.; Shah, R.; Patel, S. \& Economou, S. G. Pheochromocytoma: Operative Strategy. Surgery, 98(5):927-30, 1985.

Fernández-Cruz, L. Laparoscopic adrenal surgery. Br. J. Surg., 83(6):7213, 1996.

Gagner, M.; Pomp, A.; Heniford, B. T.; Pharand, D. \& Lacroix, A. Laparoscopic adrenalectomy: lessons learned from 100 consecutive procedures. Ann. Surg., 226(3):238-47, 1997.

Gaunt, R. History of the Adrenal Cortex. In: Blaschko, H. K. F.; Sayers, G. \& Smith, A. D. Handbook of Physiology, Sect. 7. Endocrinology. Vol VI: Adrenal Gland. Washington D. C., American Physiological Society, 1975.

Hamidi, C.; Göya, C.; Hattapoglu, S.; Çetinçakmak, M. G.; Teke, M.; Dusak, A. \& Kuday, S. Adrenal bez kalınlıgının multi-dedektörlü bilgisayarlı tomografi ile degerlendirilmesi. J. Clin. Exp. Investig., 3(3):392-4, 2012.

Harrison, T. S. Adrenal Glands. In: Schwartz, S. I. (Ed.). Principles of Surgery. 4th ed. McGraw Hill Book Co., Singapore, 1985. pp.1488.

Karstaedt, N.; Sagel, S. S.; Stanley, R. J.; Melson, G. L. \& Levitt, A. G. Computed tomography of the adrenal gland. Radiology, 129(3):72330, 1978.

Konus $_{s}$, O. L.; Ozdemir, A.; Akkaya, A.; Erbas s, G.; Celik, H. \& Is sik, S. Normal liver, spleen, and kidney dimensions in neonates, infants, and children: evaluation with sonography. A. J. R. Am. J. Roentgenol., 171(6):1693-8, 1998.

Miekos, E. Anatomical basis of radiodiagnosis of the adrenal gland. Int. Urol. Nephrol., 11(3):193-200, 1979.

Montagne, J. P.; Kressel, H. Y.; Korobkin, M. \& Moss, A. A. Computed tomography of the normal adrenal glands. A. J. R. Am. J. Roentgenol., 130(5):963-6, 1978

Montero, F.; Terzolo, M.; Amaldi, G.; Osella, G.; Masini, A. M.; Ali, A.; Giovagnetti, M.; Opocher, G. \& Angeli, A. A survey on adrenal incidentaloma in Italy. Study Group on Adrenal Tumors of the Italian Society of Endocrinology. J. Clin. Endocrinol. Metab., 85(2):637-44, 2000

Moore, K. L. \& Persaud, T. V. N. The Developing Human: Clinically Oriented Embryology. $5^{\text {th }}$ ed. Philadelphia, W. B. Saunders, 1993.

Schneller, J.; Reiser, M.; Beuschlein, F.; Osswald, A.; Pallauf, A.; Riester, A.; Tietze, J. K.; Reincke, M. \& Degenhart, C. Linear and volumetric evaluation of the adrenal gland--MDCT-based measurements of the adrenals. Acad. Radiol., 21(11):1465-74, 2014.

Tannenbaum, M. Ultrastructural Pathology of the Adrenal Cortex. In: Sommers, S. C. (Ed.). Endocrine Pathology Deccermial. New York, Appleton-Century-Crofts, 1975. pp.423.

Vincent, J. M.; Morrison, I. D.; Armstrong, P. \& Reznek, R. H. The size of normal adrenal glands on computed tomography. Clin. Radiol., 49(7):453-5, 1994.

Wang, X.; Jin, Z. Y.; Xue, H. D.; Liu, W.; Sun, H.; Chen, Y. \& Xu, K. 2013. Evaluation of normal adrenal gland volume by 64-Slice CT. Chin. Med. Sci. J., 27(4):220-4, 2013.

Wells, S. A. J. \& Santen, R. J. The Pituitary and adrenal glands. In: Sabiston, D. C. (Ed.). Textbook of Surgery. 13 ${ }^{\text {th }}$ ed. W. B. Saunders Co., 1986.

\section{Corresponding Author:}

Duygu Akin

Department of Anatomy

Meram Faculty of Medicine

Necmettin Erbakan University

Konya

TURKEY

E-Mail: d.akin.42@hotmail.com

Received: 03-05-2016

Accepted: 20-12-2016 